\title{
Observers for Interconnected Nonlinear and Linear Systems *
}

\author{
Håvard Fjær Grip ${ }^{\text {a }}$, Ali Saberi ${ }^{\mathrm{a}}$, and Tor A. Johansen ${ }^{\mathrm{b}}$ \\ ${ }^{a}$ School of Electrical Engineering and Computer Science, Washington State University, Pullman, WA 99164, USA \\ ${ }^{\mathrm{b}}$ Department of Engineering Cybernetics, Norwegian University of Science and Technology, 7491 Trondheim, Norway
}

\begin{abstract}
In this paper we begin by assuming that an observer with a corresponding quadratic-type Lyapunov function has been designed for a given nonlinear system. We then consider the problem that arises when the output of that nonlinear system is not directly available; instead, it acts as an input to a second, linear system from which a partial-state measurement is in turn available. We develop an observer design methodology for the resulting cascade interconnection, based on estimating the unavailable output together with the states of the linear system. We also extend this methodology to a more general class of feedback-interconnected systems. Under a set of technical assumptions, the overall error dynamics is proven to be globally exponentially stable if the gains are chosen to satisfy an $\mathscr{H}_{\infty}$ condition. We illustrate application of the methodology by considering a navigation example based on integration of inertial and satellite measurements.
\end{abstract}

Key words: Nonlinear observer and filter design; Interconnected systems; Guidance, navigation, and control of vehicles

\section{Introduction}

Over the past decades, the topic of nonlinear state estimation has been extensively treated in the literature, and many different design methodologies have been developed. These include stochastic techniques such as the extended and unscented Kalman filter (see, e.g., Brown and Hwang, 1997; Julier and Uhlmann, 2004) and the particle filter (e.g., Djurić et al., 2003); the use of nonlinear state transformations to achieve linear error dynamics (Krener and Isidori, 1983; Marino and Tomei, 1995); the use of linear observer dynamics in combination with a nonlinear transformation (Kazantis and Kravaris, 1998); design of observer gains to achieve robustness against Lipschitz continuous nonlinearities using, for example, LMIs or Riccati equations (see Thau, 1973; Rajamani, 1998; Zemouche, Boutayeb, and Bara, 2008; Phanomchoeng and Rajamani, 2010); the application of high gain to suppress Lipschitz continuous nonlinearities, both for left-invertible systems (Esfandiari and Khalil, 1987; Saberi and Sannuti, 1990), and non-left-invertible systems (e.g., Gauthier, Hammouri, and Othman, 1992; Bornard and Hammouri, 2002; Grip and Saberi, 2010); the exploitation of monotonic nonlinearities (Arcak and Kokotović, 2001; Fan and Arcak, 2003), or more general nonlinearities satisfying incremental quadratic

\footnotetext{
* The work of Håvard Fjær Grip is supported by the Research Council of Norway. The work of Ali Saberi is partially supported by NAVY grants ONR KKK777SB001 and ONR KKK760SB0012.
}

constraints (Açıkmeşe and Corless, 2011); sliding observers (Slotine, Hedrick, and Misawa, 1987; Drakunov, 1992); and moving-horizon estimation (Moraal and Grizzle, 1995). This list is by no means exhaustive, and in addition to general methodologies, application-specific designs proliferate throughout the literature.

In those cases where stability of the observation error can be explicitly proven, the main tool for doing so is often a Lyapunov function, and very often this Lyapunov function is of a quadratic type. Specifically, many continuoustime designs enable the construction of a Lyapunov function $V(t, \tilde{x})$ with the properties that $\alpha_{1}\|\tilde{x}\|^{2} \leq V(t, \tilde{x}) \leq \alpha_{2}\|\tilde{x}\|^{2}$, $\dot{V}(t, \tilde{x}) \leq-\alpha_{3}\|\tilde{x}\|^{2}$, and $\|[\partial V / \partial \tilde{x}](t, \tilde{x})\| \leq \alpha_{4}\|\tilde{x}\|$, where $\tilde{x}$ is the observation error variable (e.g., Krener and Isidori, 1983; Marino and Tomei, 1995; Rajamani, 1998; Zemouche et al., 2008; Phanomchoeng and Rajamani, 2010; Esfandiari and Khalil, 1987; Saberi and Sannuti, 1990; Gauthier et al., 1992; Bornard and Hammouri, 2002; Grip and Saberi, 2010; Arcak and Kokotović, 2001; Fan and Arcak, 2003; Açıkmeşe and Corless, 2011). This is not surprising, given that many designs are based at least in part on linear theory, which yields quadratic-type Lyapunov functions.

In this paper we assume that an observer with a corresponding quadratic-type Lyapunov function has already been designed for a given nonlinear system. We then consider the problem that arises when the output from that nonlinear system is not available directly, but instead available via a second, linear system. That is, the output from the nonlinear 


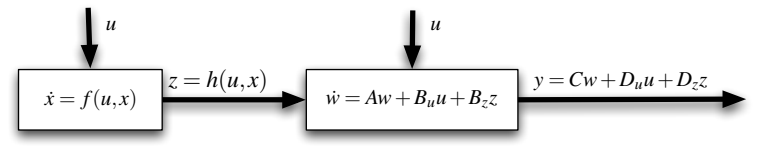

Fig. 1. Nonlinear and linear systems in cascade interconnection

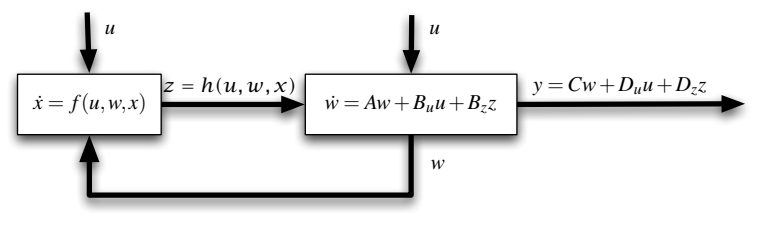

Fig. 2. Nonlinear and linear systems in feedback interconnection

system acts as the input to a linear system, from which a partial-state measurement is in turn available. This situation results in a cascade interconnection that is illustrated in Fig. 1 , and it is described by the system equations

$$
\begin{aligned}
& \Sigma_{1}:\left\{\begin{array}{l}
\dot{x}=f(u, x), \\
z=h(u, x),
\end{array}\right. \\
& \Sigma_{2}:\left\{\begin{array}{l}
\dot{w}=A w+B_{u} u+B_{z} z, \\
y=C w+D_{u} u+D_{z} z,
\end{array}\right.
\end{aligned}
$$

where $u$ is a vector of known time-varying signals, such as control inputs, reference signals, measured disturbances, or nonlinear functions of the output $y$. The partitioning of the overall system into two subsystems may stem from inherent physical divisions in a particular application, or it may be a convenient way to represent a system for design purposes. We also consider a more general case, where the state of the linear system affects the nonlinear system, resulting in the feedback interconnection illustrated in Fig. 2. In this case, the nonlinear subsystem $\Sigma_{1}$ is replaced by

$$
\Sigma_{1}^{*}:\left\{\begin{array}{l}
\dot{x}=f(u, w, x), \\
z=h(u, w, x) .
\end{array}\right.
$$

Our problem formulation is partly inspired by our main example in Section 5, which deals with estimation of position, velocity, and attitude using integrated satellite (GNSS) and inertial (INS) measurements. This design is of interest in its own right, as it provides a conceptually simple and extensible solution with global stability proofs.

\subsection{Relationship to Previous Work}

The main idea behind our design is simple: since an observer is already available for the nonlinear $\Sigma_{1}$ subsystem with output $z$, we try to implement that observer using an estimate of $z$, denoted by $\hat{z}$. In order to produce such an estimate, we extend the state space of the linear subsystem $\Sigma_{2}$ to include $z$ as an additional state, and construct an observer for this extended system.

The idea of extending the state space to obtain estimates of system inputs is not new. In particular, high-gain designs with an extended state space have been employed recently by Freidovich and Khalil $(2007,2008)$ for monitoring the decrease of Lyapunov functions and for transient performance recovery; and by the authors for the purpose of nonlinear parameter estimation (Grip, Saberi, and Johansen, 2011b).

Our design methodology, being sequential in nature, is reminiscent of recursive observer design methodologies, where an observer is designed in stages for a chain of interconnected subsystems. We point in particular to the work of Shim and Seo (2003), who treat systems similar to our feedback-interconnected case, but where the $\Sigma_{2}$ subsystem can be viewed as a chain of integrators with added nonlinearities in a lower-triangular form. In this paper we do not permit nonlinearities in $\Sigma_{2}$ (except those that depend only on known signals, and which can therefore be incorporated into $u$ ). However, the class of linear $\Sigma_{2}$ subsystems covered by our formulation is significantly larger than that of Shim and Seo (2003). More importantly, the design of Shim and Seo (2003) ensures stability through a fairly complicated multi-stage procedure that leaves little room for performance considerations, whereas our design only requires the construction of linear gains to ensure that the $\mathscr{H}_{\infty}$ norm of a particular transfer matrix is sufficiently small. Finally, we do not impose the same restrictions on the injection term of the $\Sigma_{1}$ observer.

The observer design for cascade interconnections has previously been presented in conference form (Grip, Saberi, and Johansen, 2011c).

\subsection{Preliminaries}

We denote by $\mathbb{R}_{>0}$ the nonnegative real numbers. For a vector or matrix $X, X^{\prime}$ denotes its transpose. The operator $\|\cdot\|$ denotes the Euclidean norm for vectors and the Frobenius norm for matrices. For a symmetric positive-semidefinite matrix $P$, the maximum and minimum eigenvalues are denoted by $\lambda_{\max }(P)$ and $\lambda_{\min }(P)$. Throughout the paper we assume that all signals are sufficiently smooth to allow differentiation when necessary. For a multivariable function $F(\chi)$, $\chi=\left(\chi_{1}, \ldots, \chi_{n}\right)$, we write $\partial_{\chi_{i}} F(\chi)=\left[\partial F / \partial \chi_{i}\right](\chi)$. When considering systems of the form $\dot{\chi}=F(t, \chi)$, we assume that all functions involved are sufficiently smooth to guarantee that $F(t, \chi)$ is piecewise continuous in $t$ and locally Lipschitz continuous in $\chi$, uniformly in $t$, on $\mathbb{R}_{\geq 0} \times \mathbb{R}^{n}$. The solution of this system, initialized at time $t=0$ with initial condition $\chi(0)$ is denoted by $\chi(t)$. To simplify notation, we omit function arguments when possible.

\section{Initial Assumptions}

We start by considering the system (1), where $x \in \mathbb{R}^{n_{x}}, u \in$ $\mathbb{R}^{m}, z \in \mathbb{R}^{p_{z}}, w \in \mathbb{R}^{n_{w}}$, and $y \in \mathbb{R}^{p_{y}}$. Note that the system may have been transformed from its original coordinate basis in order to be better suited for observer design. We assume that 
an observer has already been constructed for the system $\Sigma_{1}$, on the form

$$
\dot{\hat{x}}=f(u, \hat{x})+g(u, \hat{x}, z) .
$$

This design could be implemented if $z$ were available, yielding the error dynamics

$$
\dot{\tilde{x}}=e(t, \tilde{x})
$$

where $\tilde{x}:=x-\hat{x}$ and $e(t, \tilde{x}):=f(u(t), x(t))-f(u(t), x(t)-$ $\tilde{x})-g(u(t), x(t)-\tilde{x}, z(t))$.

Assumption 1 There exists a continuously differentiable function $V: \mathbb{R}_{\geq 0} \times \mathbb{R}^{n_{x}} \rightarrow \mathbb{R}_{\geq 0}$ and positive constants $\alpha_{1}, \ldots, \alpha_{4}$ such that for all $(t, \tilde{x}) \in \mathbb{R}_{\geq 0} \times \mathbb{R}^{n_{x}}$,

$$
\begin{gathered}
\alpha_{1}\|\tilde{x}\|^{2} \leq V(t, \tilde{x}) \leq \alpha_{2}\|\tilde{x}\|^{2}, \\
\frac{\partial V}{\partial t}(t, \tilde{x})+\frac{\partial V}{\partial \tilde{x}}(t, \tilde{x}) e(t, \tilde{x}) \leq-\alpha_{3}\|\tilde{x}\|^{2}, \\
\left\|\frac{\partial V}{\partial \tilde{x}}(t, \tilde{x})\right\| \leq \alpha_{4}\|\tilde{x}\| .
\end{gathered}
$$

Assumption 1 ensures exponential stability of (4). Because we will eventually replace $z$ in (3) with an estimate $\hat{z}$, we need an assumption regarding the sensitivity of $g(u, \hat{x}, z)$ to changes in $z$.

Assumption 2 There exists an $L_{1}>0$ such that for all $(t, \hat{x}, \hat{z}) \in \mathbb{R}_{\geq 0} \times \mathbb{R}^{n_{x}} \times \mathbb{R}^{p_{z}},\|g(u(t), \hat{x}, z(t))-g(u(t), \hat{x}, \hat{z})\| \leq$ $L_{1}\|z(t)-\hat{z}\|$.

For our analysis it is convenient to define a function

$$
d(u, \dot{u}, x)=\frac{\partial h}{\partial u}(u, x) \dot{u}+\frac{\partial h}{\partial x}(u, x) f(u, x),
$$

corresponding to the time derivative of the output $z$.

Assumption 3 There exists an $L_{2}>0$ such that for all $(t, \hat{x}) \in \mathbb{R}_{\geq 0} \times \mathbb{R}^{n_{x}},\|d(u(t), \dot{u}(t), x(t))-d(u(t), \dot{u}(t), \hat{x})\| \leq$ $L_{2}\|x(t)-\hat{x}\|$.

Remark 1 Assumptions 2 and 3 specify global Lipschitztype conditions on the functions $g$ and $d$. These conditions are restrictive, but can be made far less so if $u, \dot{u}, x$, and $z$ have known bounds, which is usually the case in physical estimation problems. An explanation of how this boundedness can be exploited-by introducing saturations on the arguments of $f, h$, and $g-$ is given in Appendix A.

Our final assumption concerns the linear system $\Sigma_{2}$.

Assumption 4 The pair $(A, C)$ is detectable and the quadruple $\left(A, B_{z}, C, D_{z}\right)$ is left-invertible with no invariant zeros at the origin.

Left-invertibility of a linear system means that two trajectories originating from the same initial condition will produce identical outputs for all $t \geq 0$ only if the inputs are also identical (see, e.g., Saberi, Stoorvogel, and Sannuti, 2006,
Ch. 3.2.2). For example, every SISO system is left-invertible (unless its transfer function is identically zero).

We shall also assume, without loss of generality, that the matrices $\left[B_{z}^{\prime}, D_{z}^{\prime}\right]^{\prime}$ and $\left[C, D_{z}\right]$ are of maximal rank $p_{z}$ and $p_{y}$, respectively (i.e., there are no redundant elements of $z$ and the elements of $y$ are linearly independent). If this assumption does not hold, it is easily satisfied by redefining $z$ or $y$ to eliminate redundancies.

\section{Observer Design}

We now present a design methodology for the overall system. We start by introducing an extended version of the linear system $\Sigma_{2}$, which includes $z$ as an additional state. The extended system vector is given by $w_{\mathrm{e}}=\left[w^{\prime}, z^{\prime}\right]^{\prime}$, and the dynamics of the extended system is given by

$$
\begin{aligned}
\dot{w}_{\mathrm{e}} & =\mathfrak{A}_{w_{\mathrm{e}}}+\mathfrak{B}_{u} u+\mathfrak{B}_{d} d(u, \dot{u}, x), \\
y & =\mathfrak{C} w_{\mathrm{e}}+D_{u} u,
\end{aligned}
$$

$$
\mathfrak{A}=\left[\begin{array}{cc}
A & B_{z} \\
0 & 0
\end{array}\right], \mathfrak{B}_{u}=\left[\begin{array}{c}
B_{u} \\
0
\end{array}\right], \mathfrak{B}_{d}=\left[\begin{array}{l}
0 \\
I
\end{array}\right], \mathfrak{C}=\left[\begin{array}{ll}
C & D_{z}
\end{array}\right] .
$$

We define an observer for the extended system in the following way:

$$
\begin{aligned}
\dot{\hat{w}} & =A \hat{w}+B_{u} u+B_{z} \hat{z}+K_{w}\left(y-C \hat{w}-D_{u} u-D_{z} \hat{z}\right), \\
\dot{\xi} & =-\frac{\partial h}{\partial x}(u, \hat{x}) g(u, \hat{x}, \hat{z})+K_{z}\left(y-C \hat{w}-D_{u} u-D_{z} \hat{z}\right) \\
\hat{z} & =h(u, \hat{x})+\xi
\end{aligned}
$$

where $K_{w}$ and $K_{z}$ are observer gains to be determined. The variables $\hat{w}$ and $\hat{z}$ are estimates of $w$ and $z$, and they are gathered in a vector $\hat{w}_{\mathrm{e}}=\left[\hat{w}^{\prime}, \hat{z}^{\prime}\right]^{\prime}$. It is convenient to analyze the observer with respect $\hat{w}_{\mathrm{e}}$, which constitutes a nonsingular transformation from the original observer states $(\hat{w}, \xi)$.

In addition to the observer (9), we implement the already existing observer for the system $\Sigma_{1}$; however, we do so not based on $z$, but the estimate $\hat{z}$ :

$$
\dot{\hat{x}}=f(u, \hat{x})+g(u, \hat{x}, \hat{z})
$$

By differentiating $\hat{z}$ and defining $K=\left[K_{w}^{\prime}, K_{z}^{\prime}\right]^{\prime}$, we can now calculate the dynamics of $\hat{w}_{\mathrm{e}}$ as

$$
\dot{\hat{w}}_{\mathrm{e}}=\mathfrak{A} \hat{w}_{\mathrm{e}}+\mathfrak{B}_{u} u+\mathfrak{B}_{d} d(u, \dot{u}, \hat{x})+K\left(y-\mathfrak{C} \hat{w}_{\mathrm{e}}-D_{u} u\right) .
$$

\subsection{Stability}

The dynamics of the estimation errors $\tilde{w}_{\mathrm{e}}:=w_{\mathrm{e}}-\hat{w}_{\mathrm{e}}$ and $\tilde{x}$ can be written as

$$
\dot{\tilde{w}}_{\mathrm{e}}=(\mathfrak{A}-K \mathfrak{C}) \tilde{w}_{\mathrm{e}}+\mathfrak{B}_{d} \tilde{d}(t, \tilde{x})
$$




$$
\dot{\tilde{x}}=e(t, \tilde{x})+g(u, \hat{x}, z)-g(u, \hat{x}, \hat{z}) .
$$

where $\tilde{d}(t, \tilde{x}):=d(u, \dot{u}, x)-d(u, \dot{u}, x-\tilde{x})$. Our goal is to choose an observer gain matrix $K$ to stabilize the error dynamics. Toward this end, we define

$$
H(s)=(I s-\mathfrak{A}+K \mathfrak{C})^{-1} \mathfrak{B}_{d},
$$

which is the transfer matrix from the input point of $\tilde{d}(t, \tilde{x})$ in (12a) to the error $\tilde{w}_{\mathrm{e}}$. The following result is proven in Appendix B.

Theorem 1 If $K$ is chosen such that $\mathfrak{A}-K \mathfrak{C}$ is Hurwitz and $\|H(s)\|_{\infty}<\gamma:=4 \alpha_{3} /\left(4 L_{2}^{2}+\alpha_{4}^{2} L_{1}^{2}\right)$, then the error dynamics (12) is globally exponentially stable.

Having established a sufficient condition for stability in terms of the gain $K$, the next question is whether there exists a gain that satisfies the condition. This question is answered by the following theorem, which is proven in Appendix B.

Theorem 2 There exists $a \gamma^{*}>0$ such that, for all $\gamma>\gamma^{*}, K$ can be chosen such that $\mathfrak{A}-K \mathfrak{C}$ is Hurwitz and $\|H(s)\|_{\infty}<\gamma$. Furthermore, if the quadruple $\left(A, B_{z}, C, D_{z}\right)$ is minimumphase, then $\gamma^{*}=0$.

According to Theorem 2, we can design $K$ so that $\|H(s)\|_{\infty}$ comes arbitrarily close to some lower limit $\gamma^{*} \geq 0$. In general, this lower limit may not be small enough to satisfy Theorem 1. However, if we impose an additional minimumphase condition on $\Sigma_{2}$, then the lower limit is $\gamma^{*}=0$, meaning that the conditions of Theorem 1 can always be satisfied.

\subsection{Gain Synthesis and Tuning}

Although Theorem 1 enables us to compute an explicit numerical value of $\gamma$ such that $\|H(s)\|_{\infty}<\gamma$ ensures stability, such a computation is likely to be conservative and lead to poor performance. It is therefore preferable in practice to tune the observer by starting with a large value of $\gamma$ and decreasing it gradually until the desired stability and performance is achieved. As a practical matter, ensuring that $\mathfrak{A}-K \mathfrak{C}$ is Hurwitz and that $\|H(s)\|_{\infty}<\gamma$ can be achieved using several different $\mathscr{H}_{\infty}$ design methods; specifically, Riccati-based methods, direct methods, and LMI-based methods (see Saberi et al., 2006).

The use of LMIs is attractive, because it allows for easy incorporation of additional performance criteria in the design process. For a given $\gamma$, it follows from the boundedreal lemma (see, e.g., Saberi et al., 2006, Th. 11.45) that $\|H(s)\|_{\infty}<\gamma$ is satisfied by choosing $K=P^{-1} X$, where $X$ and $P=P^{\prime}>0$ are solutions of the LMI

$$
\left[\begin{array}{cr}
P \mathfrak{A}+\mathfrak{A}^{\prime} P-X \mathfrak{C}-\mathfrak{C}^{\prime} X^{\prime}+I & P \mathfrak{B}_{d} \\
\mathfrak{B}_{d}^{\prime} P & -\gamma^{2} I
\end{array}\right]<0 .
$$

The solution of this LMI is far from unique-there are additional degrees of freedom in choosing $K$ that can be used to improve performance. In particular, it was shown by Chilali and Gahinet (1996) that by including additional LMIs based on a common Lyapunov matrix $P$, it is possible to constrain the closed-loop poles to some convex LMI region (assuming the region is feasible for the given $\mathscr{H}_{\infty}$ objective), or to incorporate additional $\mathscr{H}_{\infty}$ or $\mathscr{H}_{2}$ minimization objectives.

Of particular concern when designing observers is the effect of measurement noise. Suppose that $y$ is affected by additive noise $n$; that is, $y=C w+D_{u} u+D_{z} z+N n$. The transfer matrix from the input point of $n$ to $\tilde{w}_{\mathrm{e}}$ is $G(s):=$ $-(s I-\mathfrak{A}+K \mathfrak{C})^{-1} K N$. We can limit the effect of the measurement noise on $\tilde{w}_{\mathrm{e}}$ by minimizing a bound on $\|G(s)\|_{\infty}$, while at the same time ensuring that (14) is satisfied. This is done by minimizing a value $\gamma_{n}^{2}>0$ subject to the LMIs (14) and

$$
\left[\begin{array}{cr}
P \mathfrak{A}+\mathfrak{A}^{\prime} P-X \mathfrak{C}-\mathfrak{C}^{\prime} X^{\prime}+I & -X N \\
-N^{\prime} X^{\prime} & -\gamma_{n}^{2} I
\end{array}\right]<0 .
$$

The LMI (15) ensures that $\|G(s)\|_{\infty} \leq \gamma_{n}^{2}$.

As in other $\mathscr{H}_{\infty}$-based design problems, it may be beneficial to pre-scale the various input and output channels of the transfer function to the same order of magnitude.

In most cases, one also has the freedom to adjust gains in the observer for the $\Sigma_{1}$ subsystem. Such adjustments will affect the performance of the overall observer and the best choice of gain $K$, in ways that are difficult to characterize precisely. In general, a certain amount of tuning based on trial and error is needed to find the best combination of gains for the overall observer.

\section{Feedback-Interconnected Systems}

We now consider the more general problem formulation depicted in Fig. 2, where the $\Sigma_{1}$ subsystem is replaced by a subsystem $\Sigma_{1}^{*}$ that is affected by the linear system $\Sigma_{2}$. We start by adjusting some of our initial assumptions. Instead of (3), we now assume that an observer already exists for the system $\Sigma_{1}^{*}$, on the form

$$
\dot{\hat{x}}=f(u, w, \hat{x})+g(u, \hat{x}, w, z) .
$$

This results in error dynamics of the form (4), where $e(t, \tilde{x}):=f(u(t), w(t), x(t))-f(u(t), w(t), x(t)-\tilde{x})-$ $g(u(t), x(t)-\tilde{x}, w(t), z(t))$. We assume that Assumption 1 holds for this redefined version of $e(t, \tilde{x})$. Assumption 2 is restated as follows:

Assumption 2' There exists an $L_{1}>0$ such that for all $(t, \hat{x}, \hat{w}, \hat{z}) \in \mathbb{R}_{\geq 0} \times \mathbb{R}^{n_{x}} \times \mathbb{R}^{n_{w}} \times \mathbb{R}^{p_{z}}, \quad \| f(u(t), w(t), \hat{x})-$ $f(u(t), \hat{w}, \hat{x})+g(u(t), \hat{x}, w(t), z(t))-g(u(t), \hat{x}, \hat{w}, \hat{z}) \| \leq$ $L_{1}\left\|\left[w^{\prime}(t)-\hat{w}^{\prime}, z^{\prime}(t)-\hat{z}^{\prime}\right]^{\prime}\right\|$. 
The function $d$ is redefined as

$$
\begin{aligned}
d(u, \dot{u}, w, z, x)= & \frac{\partial h}{\partial u}(u, w, x) \dot{u}+\frac{\partial h}{\partial x}(u, w, x) f(u, w, x) \\
& +\frac{\partial h}{\partial w}(u, w, x)\left(A w+B_{u} u+B_{z} z\right)
\end{aligned}
$$

which represents the time derivative of $h(u, w, x)$. Assumption 3 is restated as follows:

Assumption $3^{\prime}$ There exists an $L_{2}>0$ such that for all $(t, \hat{w}, \hat{x}) \in \mathbb{R}_{\geq 0} \times \mathbb{R}^{n_{w}} \times \mathbb{R}^{n_{x}}, \| d(u(t), \dot{u}(t), w(t), z(t), x(t))-$ $d(u(t), \dot{u}(t), \hat{w}, \hat{z}, \hat{x})\left\|\leq L_{2}\right\|\left[w^{\prime}(t)-\hat{w}^{\prime}, z^{\prime}(t)-\hat{z}^{\prime}, x^{\prime}(t)-\hat{x}^{\prime}\right]^{\prime} \|$.

Remark 2 Similar to Assumptions 2 and 3, Assumptions $2^{\prime}$ and $3^{\prime}$ can be relaxed if $u, \dot{u}, x, z$, and $w$ belong to compact sets, by using saturation techniques.

Assumption 4 remains the same as before.

\subsection{Observer and Stability}

For the feedback-interconnected system, we define an observer for the extended system vector $w_{\mathrm{e}}=\left[w^{\prime}, z^{\prime}\right]^{\prime}$ as

$$
\begin{aligned}
\hat{\hat{w}}= & A \hat{w}+B_{u} u+B_{z} \hat{z}+K_{w}\left(y-C \hat{w}-D_{u} u-D_{z} \hat{z}\right), \\
\dot{\xi}= & -\frac{\partial h}{\partial x}(u, \hat{w}, \hat{x}) g(u, \hat{x}, \hat{w}, \hat{z}) \\
& -\frac{\partial h}{\partial w}(u, \hat{w}, \hat{x}) K_{w}\left(y-C \hat{w}-D_{u} u-D_{z} \hat{z}\right), \\
& +K_{z}\left(y-C \hat{w}-D_{u} u-D_{z} \hat{z}\right), \\
\hat{z}= & h(u, \hat{w}, \hat{x})+\xi .
\end{aligned}
$$

The observer for the $\Sigma_{1}^{*}$ subsystem is defined based on the estimates $\hat{w}$ and $\hat{z}$ :

$$
\dot{\hat{x}}=f(u, \hat{w}, \hat{x})+g(u, \hat{x}, \hat{w}, \hat{z}) .
$$

By differentiating $\hat{z}$ it is easily verified that the dynamics of $\hat{w}_{\mathrm{e}}$ is described by

$$
\begin{aligned}
\dot{\hat{w}}_{\mathrm{e}}= & \mathfrak{A} \hat{w}_{\mathrm{e}}+\mathfrak{B}_{u} u+\mathfrak{B}_{d} d(u, \dot{u}, \hat{w}, \hat{z}, \hat{x}) \\
& +K\left(y-\mathfrak{C} \hat{w}_{\mathrm{e}}-D_{u} u\right) .
\end{aligned}
$$

The dynamics of $\tilde{w}_{\mathrm{e}}$ and $\tilde{x}$ is therefore described by

$$
\begin{aligned}
\dot{\tilde{w}}_{\mathrm{e}}= & (\mathfrak{A}-K \mathfrak{C}) \tilde{w}_{\mathrm{e}}+\mathfrak{B}_{d} \tilde{d}(t, \tilde{w}, \tilde{z}, \tilde{x}) \\
\dot{\tilde{x}}= & e(t, \tilde{x})+f(u, w, \hat{x})-f(u, \hat{w}, \hat{x}) \\
& +g(u, \hat{x}, w, z)-g(u, \hat{x}, \hat{w}, \hat{z})
\end{aligned}
$$

where $\tilde{d}(t, \tilde{w}, \tilde{z}, \tilde{x}):=d(u, \dot{u}, w, z, x)-d(u, \dot{u}, w-\tilde{w}, z-\tilde{z}, x-$ $\tilde{x})$. We can now state the equivalent of Theorem 1 for the feedback-interconnected system.

Theorem $\mathbf{1}^{\prime}$ There exists a $\gamma>0$ such that, if $K$ is chosen such that $\mathfrak{A}-K \mathfrak{C}$ is Hurwitz and $\|H(s)\|_{\infty}<\gamma$, then the error dynamics (20) is globally exponentially stable.
The proof of Theorem 1' is given in Appendix B. As before, it follows from Theorem 2 that the conditions of Theorem $1^{\prime}$ can always be satisfied if the quadruple $\left(A, B_{z}, C, D_{z}\right)$ is minimum-phase.

Example 1 Consider the nonlinear system

$$
\Sigma_{1}: \dot{x}=\left[\begin{array}{cc}
0 & 1 \\
1 & -1
\end{array}\right] x+\Phi(t, x), \quad z=\left[\begin{array}{ll}
0 & 1
\end{array}\right] x,
$$

where $\Phi(t, x)$ has Lipschitz constant 0.4. Rajamani (1998) demonstrated that an observer based on a linear output injection term with gain $L \approx[69.6,11.6]^{\prime}$ stabilizes the corresponding error dynamics. Suppose instead that only a filtered output $y=1 /\left(s^{2}+s+1\right) z$ is available from this system, which can be described by a second system:

$$
\Sigma_{2}: \dot{w}=\left[\begin{array}{cc}
0 & 1 \\
-1 & -1
\end{array}\right] w+\left[\begin{array}{l}
0 \\
1
\end{array}\right] z, \quad y=\left[\begin{array}{ll}
1 & 0
\end{array}\right] w .
$$

The method of Rajamani (1998) does not find a solution when applied to the overall system with this state-space representation. It is nevertheless trivial to confirm that $\Sigma_{1}$ and the original observer satisfies Assumptions 1-3; and that $\Sigma_{2}$ satisfies Assumption 4 and is minimum-phase. Hence, we can apply our methodology based on the original observer. Indeed, we can do so even if $\Phi$ is also a function of $w$.

\section{GNSS/INS Integration}

Navigation is the task of determining an object's position, velocity, or attitude by combining information from different sources. The available information varies depending on the application; however, the combination of satellite receivers, such as GPS, and inertial instruments (accelerometers and rate gyroscopes) is found in many applications, often together with additional sensors such as altimeters and magnetometers. The integration of satellite and inertial measurements, referred to as GNSS/INS integration, has been studied for several decades (see, e.g., Maybeck, 1979). Typically, the integration is based on an extended Kalman filter (EKF), but advances in low-cost sensor technology has spurred an interest in constructing nonlinear observers with lower computational complexity.

Most of the effort on nonlinear navigation observers has been directed toward the problem of estimating attitude, typically based on an explicit attitude measurement or a comparison between vectors in two coordinate frames (see Crassidis, Markley, and Cheng, 2007, for a survey). Vik and Fossen (2001) studied GNSS/INS integration, with the assumption that the attitude could be measured independently from the position and velocity. Hua (2010) did not make this assumption, and constructed algorithms based only on GNSS position and velocity together with inertial and magnetometer measurements. In this section we consider the same problem as Hua (2010), within the theoretical framework established 
in this paper. The aim is to illustrate the general methodology of this paper, and we therefore ignore some important aspects such as bias estimation and the effect of various noise sources.

The dynamics of the system is described by

$$
\dot{R}=R S\left(\omega^{\mathrm{b}}\right), \quad \dot{p}^{\mathrm{n}}=v^{\mathrm{n}}, \quad \dot{v}^{\mathrm{n}}=a^{\mathrm{n}}+g^{\mathrm{n}},
$$

where $R \in \mathrm{SO}(3)$ is a rotation matrix from the body-fixed coordinate frame to an earth-fixed reference frame, which describes the attitude; $p^{\mathrm{n}}$ and $v^{\mathrm{n}}$ are the position and velocity in the earth-fixed frame; $\omega^{\mathrm{b}}$ is the angular velocity of the body-fixed frame with respect to the earth-fixed frame, given in body-fixed coordinates; $g^{\mathrm{n}}$ is the gravity vector; and $a^{\mathrm{n}}$ is the proper acceleration in the earth-fixed coordinate frame. ${ }^{1}$ The function $S(\cdot)$ generates a skew-symmetric matrix from its argument, so that for any $x, y \in \mathbb{R}^{3}, S(x) y=x \times y$. We assume that $p^{\mathrm{n}}$ and $v^{\mathrm{n}}$ are available as measurements from the GNSS receiver. The inertial sensors provide measurements of $\omega^{\mathrm{b}}$, as well as an accelerometer measurement $a^{\mathrm{b}}$, which is related to $a^{\mathrm{n}}$ by $a^{\mathrm{n}}=R a^{\mathrm{b}}$. We furthermore assume that a magnetometer measurement $m^{\mathrm{b}}$ is available, and that the earth's magnetic field $m^{\mathrm{n}}$ is known.

\subsection{Observer}

We first consider the problem of estimating the attitude $R$, assuming for the time being that $a^{\mathrm{n}}$ is available as a measurement. Since $m^{\mathrm{n}}=R m^{\mathrm{b}}$ and $a^{\mathrm{n}}=R a^{\mathrm{b}}$, we can construct an observer based on comparing $m^{\mathrm{b}}$ with $m^{\mathrm{n}}$ and $a^{\mathrm{b}}$ with $a^{\mathrm{n}}$, as follows:

$$
\dot{\hat{R}}=\hat{R} S\left(\omega^{\mathrm{b}}\right)+\Gamma J\left(m^{\mathrm{b}}, m^{\mathrm{n}}, a^{\mathrm{b}}, a^{\mathrm{n}}, \hat{R}\right),
$$

where $\Gamma$ is a symmetric positive-definite gain matrix and $J=A_{\mathrm{n}} A_{\mathrm{b}}^{\prime}-\hat{R} A_{\mathrm{b}} A_{\mathrm{b}}^{\prime}$, with $A_{\mathrm{b}}=\left[m^{\mathrm{b}}, m^{\mathrm{b}} \times a^{\mathrm{b}}, m^{\mathrm{b}} \times\left(m^{\mathrm{b}} \times a^{\mathrm{b}}\right)\right]$ and $A_{\mathrm{n}}=\left[m^{\mathrm{n}}, m^{\mathrm{n}} \times a^{\mathrm{n}}, m^{\mathrm{n}} \times\left(m^{\mathrm{n}} \times a^{\mathrm{n}}\right)\right]$. The definition of $J$ is inspired by the TRIAD algorithm (see Shuster and Oh, 1981), which allows the attitude to be algebraically determined based on two body-fixed vector measurements and their corresponding reference vectors, provided the bodyfixed vectors are non-parallel. To ensure that this is the case, we assume that there exists a constant $c_{\mathrm{obs}}>0$ such that $\left\|m^{\mathrm{b}} \times a^{\mathrm{b}}\right\| \geq c_{\text {obs }}$. We also assume that $a^{\mathrm{b}}, \dot{a}^{\mathrm{b}}, m^{\mathrm{b}}$, and $\omega^{\mathrm{b}}$ are uniformly bounded.

The dynamics of the estimation error $\tilde{R}=R-\hat{R}$ is

$$
\dot{\tilde{R}}=\tilde{R} S\left(\omega^{\mathrm{b}}\right)-\Gamma J\left(m^{\mathrm{b}}, m^{\mathrm{n}}, a^{\mathrm{b}}, a^{\mathrm{n}}, R-\tilde{R}\right) .
$$

The following lemma is proven in Appendix B.

\footnotetext{
1 We assume here that the earth-fixed coordinate frame is inertial. For high-precision applications, the earth's rotation must also be accounted for in the model.
}

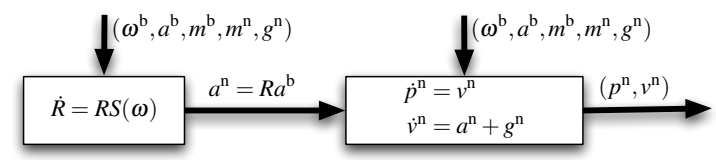

Fig. 3. Structure of system containing attitude, position, and velocity

Lemma 1 The origin of (23) is globally exponentially stable.

The observer (22) cannot be implemented, because it depends on the unmeasured variable $a^{\mathrm{n}}$. However, we see from (21) that $a^{\mathrm{n}}$ can be viewed as an input to the linear system $\dot{p}^{\mathrm{n}}=v^{\mathrm{n}}, \dot{v}^{\mathrm{n}}=a^{\mathrm{n}}+g^{\mathrm{n}}$, from which the outputs $p^{\mathrm{n}}$ and $v^{\mathrm{n}}$ are available. This situation, which is illustrated in Fig. 3, corresponds to the original problem formulation studied in this paper by defining the $\Sigma_{1}$ subsystem to contain the attitude dynamics and the $\Sigma_{2}$ subsystem to contain the position and velocity dynamics.

To enable us to treat the state of the $\Sigma_{1}$ system as a vector rather than a matrix, we alternatively write the state as $x=$ $\operatorname{vec} R$, where the vec operator stacks the columns of $R$ to form a vector (similarly, $\hat{x}=\operatorname{vec} \hat{R}$ and $\tilde{x}=\operatorname{vec} \tilde{R}$ ). We furthermore write $z=a^{\mathrm{n}}=R a^{\mathrm{b}}, w=\left[p^{\mathrm{n} \prime}, v^{\mathrm{n} \prime}\right]^{\prime}, y=\left[p^{\mathrm{n} \prime}, v^{\mathrm{n} \prime}\right]^{\prime}$, and $u=$ $\left[\omega^{\mathrm{b}^{\prime}}, a^{\mathrm{b}^{\prime}}, m^{\mathrm{b}^{\prime}}, m^{\mathrm{n}^{\prime}}, g^{\mathrm{n}}\right]^{\prime}$. The matrices $A, B_{z}, C$, and $D_{z}$ of $\Sigma_{2}$ are then given by

$$
A=\left[\begin{array}{ll}
0 & I \\
0 & 0
\end{array}\right], \quad B_{z}=\left[\begin{array}{l}
0 \\
I
\end{array}\right], \quad C=\left[\begin{array}{ll}
I & 0 \\
0 & I
\end{array}\right], \quad D_{z}=\left[\begin{array}{l}
0 \\
0
\end{array}\right] .
$$

The following lemma is proven in Appendix B.

Lemma 2 The observer (22) satisfies Assumptions 1-3. The $\Sigma_{2}$ subsystem satisfies Assumption 4 and is minimum-phase.

Since all the assumptions from Section 2 are satisfied, we can apply our design methodology by combining an observer of the form (9) with the observer (22), with $z=a^{\mathrm{n}}$ replaced by the estimate $\hat{z}$. Expanding the expression for $\hat{z}$, we write the complete observer as follows:

$$
\begin{aligned}
\dot{\hat{p}}^{\mathrm{n}}= & \hat{v}^{\mathrm{n}}+K_{p p}\left(p^{\mathrm{n}}-\hat{p}^{\mathrm{n}}\right)+K_{p v}\left(v^{\mathrm{n}}-\hat{v}^{\mathrm{n}}\right) \\
\dot{\hat{v}}^{\mathrm{n}}= & \hat{R} a^{\mathrm{b}}+\xi+g^{\mathrm{n}}+K_{v p}\left(p^{\mathrm{n}}-\hat{p}^{\mathrm{n}}\right)+K_{v v}\left(v^{\mathrm{n}}-\hat{v}^{\mathrm{n}}\right) \\
\dot{\xi}= & -\Gamma J\left(m^{\mathrm{b}}, m^{\mathrm{n}}, a^{\mathrm{b}}, \hat{R} a^{\mathrm{b}}+\xi, \hat{R}\right) a^{\mathrm{b}} \\
& +K_{z p}\left(p^{\mathrm{n}}-\hat{p}^{\mathrm{n}}\right)+K_{z v}\left(v^{\mathrm{n}}-\hat{v}^{\mathrm{n}}\right) \\
\dot{\hat{R}}= & \hat{R} S\left(\omega^{\mathrm{b}}\right)+\Gamma J\left(m^{\mathrm{b}}, m^{\mathrm{n}}, a^{\mathrm{b}}, \hat{R} a^{\mathrm{b}}+\xi, \hat{R}\right) .
\end{aligned}
$$

The gain matrix $K$, composed of $K_{p p}, K_{p v}, K_{v p}, K_{v v}, K_{z p}$, and $K_{z v}$, must be chosen to ensure stability of the observer error dynamics. This is always possible, as shown in Theorem 3 below, which follows directly from our previous results.

Theorem 3 Let $\mathfrak{A}, \mathfrak{B}_{d}$, and $\mathfrak{C}$ be defined from $A, B_{z}, C$, and $D_{z}$ according to $(8 \mathrm{c})$, and let $H(s)=(I s-\mathfrak{A}+K \mathfrak{C})^{-1} \mathfrak{B}_{d}$. There exists a $\gamma>0$ such that if $K$ is chosen such that 


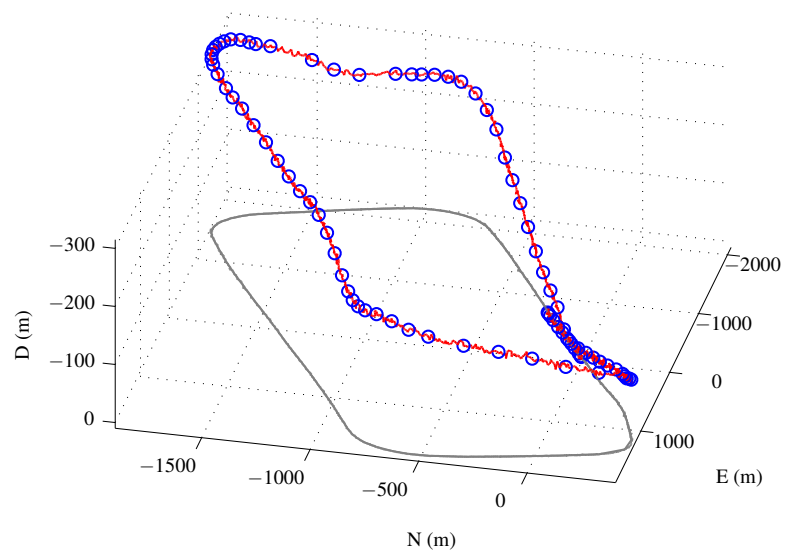

Fig. 4. True (markers) and estimated (solid) position in local North-East-Down coordinates (ground track at zero altitude shown in gray)

$\mathfrak{A}-K \mathfrak{C}$ is Hurwitz and $\|H(s)\|_{\infty}<\gamma$, then the origin of the error dynamics corresponding to the observer (24) is globally exponentially stable. Moreover, $K$ can always be chosen to satisfy these conditions.

\subsection{Simulation Results}

In order to verify the design, it is tested on simulation data from the $X$-Plane $\AA$ flight simulator. The simulated maneuver is a flight around an airport traffic pattern, from takeoff to landing, in a Cessna 172. The inertial measurements are available at $100 \mathrm{~Hz}$, and the position and velocity measurements are available at $5 \mathrm{~Hz}$. Noise has been added to the GNSS measurements.

The observer is implemented with the gain for the attitude observer set to $\Gamma=\operatorname{diag}(20,0.2,0.2)$. To make the observer robust against the GNSS measurement errors, we follow the LMI-based design strategy described in Section 3.2 with $N=$ $I$. We achieve stable estimates by choosing $\gamma=50$, which yields the gains $K_{p p} \approx 128.9 I, K_{p v} \approx 17.5 I, K_{v p} \approx 15.7 I$, $K_{v v} \approx 2.4 I, K_{z p} \approx 1.3 I$, and $K_{z v} \approx 0.2 I$. Fig. 4 shows the true and estimated position of the aircraft, and Fig. 5 shows the true velocity together with the velocity estimation error. Fig. 6 shows the true Euler angles of the attitude, together with the Euler angle estimation error (based on Euler angle estimates derived from $\hat{R}$ by inverse trigonometry).

One advantage of our design is that it can easily be adapted to changes in the linear part of the system. Suppose, for example, that the GNSS receiver only provides position measurements, which changes the linear part of the system such that $C=[I, 0]$. Assumption 4 is still satisfied in this case, and $\left(A, B_{z}, C, D_{z}\right)$ is still minimum-phase. Thus, we can apply our methodology with equal simplicity. Another possibility is to extend the attitude observer to include estimation of gyro bias, or to estimate accelerometer bias using the method of Grip, Fossen, Johansen, and Saberi (2011a), which also relies on the unmeasured variable $a^{\mathrm{n}}$.
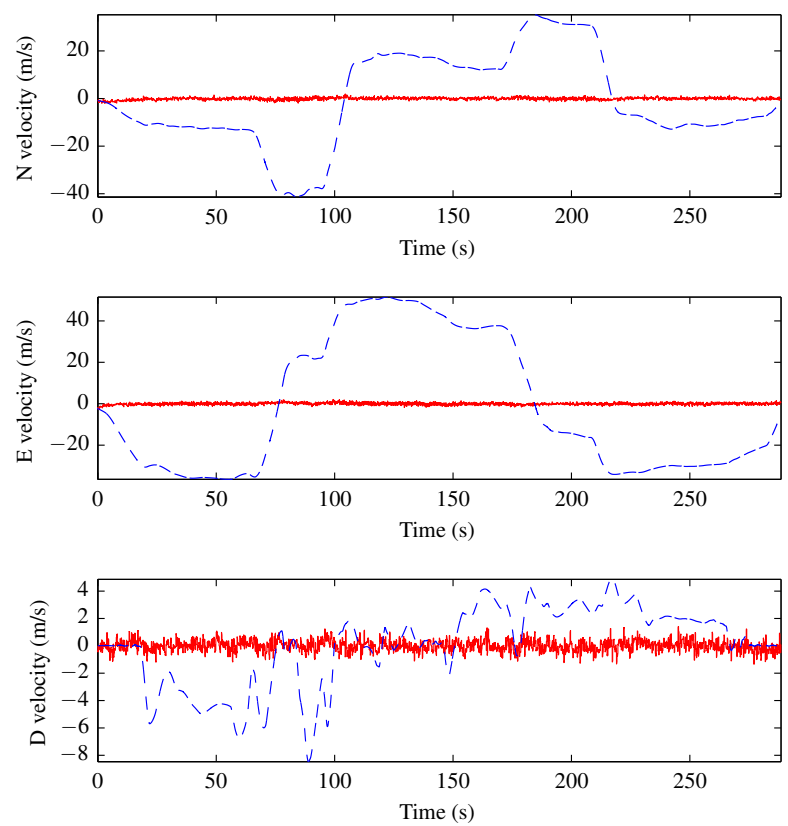

Fig. 5. True velocity (dashed) and velocity estimation error (solid) in local North-East-Down coordinates
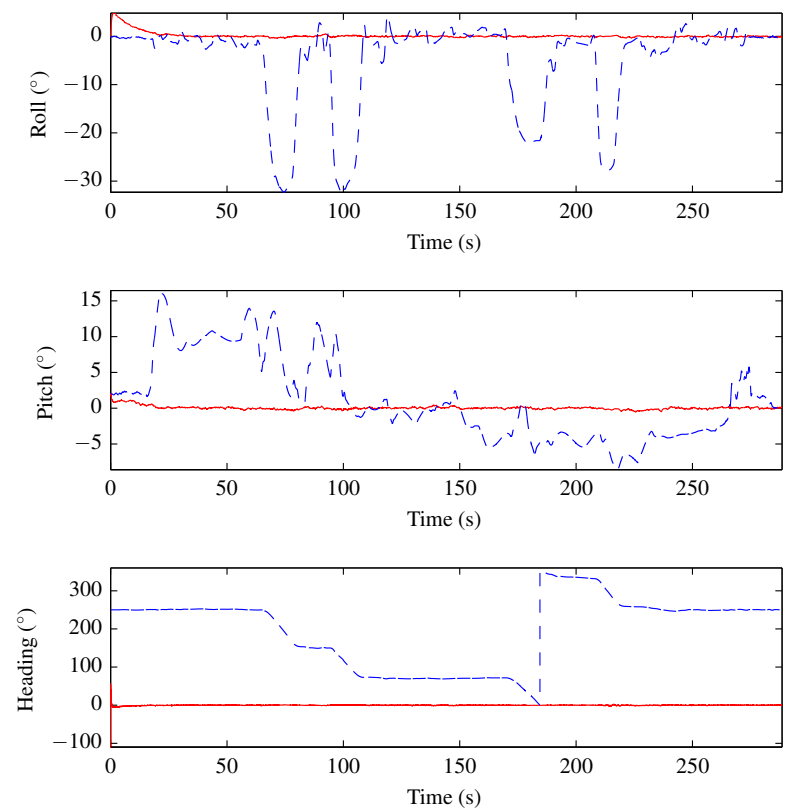

Fig. 6. True Euler angles (dashed) and Euler angle error (solid)

A disadvantage of our design is that $\hat{R}$ is in general not a rotation matrix, even though it converges to one. Thus, if one tries to extract the attitude in terms of Euler angles or quaternions from $\hat{R}$, one might temporarily have an illdefined problem. To deal with this problem it is possible to post-orthogonalize the estimate, or, alternatively, use the convergent estimate of $a^{\mathrm{n}}$ in a second estimator to produce a true rotation matrix. 


\section{Concluding Remarks}

The goal of the design methodology presented in this paper is to enable or simplify observer design for systems that are otherwise difficult to handle, by allowing the designer to focus on a smaller nonlinear subsystem. In some cases one may have the choice of applying our methodology or using a different nonlinear observer design for the overall system. The effect of such a choice on performance has not yet been investigated. However, we note that our methodology will typically result in a higher-order observer, due to the introduction of an additional state.

\section{A Satisfying the Lipschitz-Type Assumptions}

If the signals $u(t), \dot{u}(t), x(t)$, and $z(t)$ belong to compact sets $U, U^{\prime}, X$, and $Z$, then the global Lipschitz-type conditions on $g$ and $d$ can be replaced by local smoothness conditions for $f, h$, and $g$ by using saturations. This type of strategy is common in the observer literature (see, e.g., Gauthier et al., 1992). Consider first Assumption 2. Suppose that the function $g(u, \hat{x}, z)$ is locally Lipschitz continuous in $z$, uniformly in $(u, \hat{x})$, on $U \times \mathbb{R}^{n_{x}} \times \mathbb{R}^{p_{z}}$, and suppose we replace it by $g(u, \hat{x}, \operatorname{sat}(z))$, where $\operatorname{sat}(z)$ is a componentwise saturation with limits such that $\operatorname{sat}(z)=z$ for $z \in Z$. Since the saturated signal belongs to a compact set, the local Lipschitz condition implies that there exists an $L_{1}$ such that $\|g(u, \hat{x}, \operatorname{sat}(z))-g(u, \hat{x}, \operatorname{sat}(\hat{z}))\| \leq L_{1}\|\operatorname{sat}(z)-\operatorname{sat}(\hat{z})\| \leq$ $L_{1}\|z-\hat{z}\|$. Since the saturation is inactive on $Z$, the definition of $e(t, \tilde{x})$ is unaffected.

Next, consider Assumption 3. Suppose that $f(u, x)$, $\partial_{u} h(u, x)$, and $\partial_{x} h(u, x)$ are locally Lipschitz in $x$, uniformly in $u$, and suppose that we replace our model for $\Sigma_{1}$ with an alternative design model $\Sigma_{1}^{\mathrm{d}}: \dot{x}=f_{\mathrm{s}}(u, x), z=h_{\mathrm{s}}(u, x)$, where $f_{\mathrm{s}}(u, x)=f\left(u\right.$, sat $\left.^{*}(x)\right)$ and $h_{\mathrm{s}}(u, x)=h\left(u, \mathrm{sat}^{*}(x)\right)$, and sat*(.) is a smooth saturation with limits such that $\operatorname{sat}^{*}(x)=x$ for $x \in X$ (in particular, let $\partial_{x} \operatorname{sat}^{*}(\cdot)$ be bounded and Lipschitz). The design model represents an equivalent description of the physical system, since $x(t)$ never leaves the region where $\Sigma_{1}^{\mathrm{d}}=\Sigma_{1}$. With this modification, we obtain

$$
\begin{aligned}
& \|d(u, \dot{u}, x)-d(u, \dot{u}, \hat{x})\| \leq \\
& \quad\left\|\partial_{u} h\left(u, \operatorname{sat}^{*}(x)\right)-\partial_{u} h\left(u, \operatorname{sat}^{*}(\hat{x})\right)\right\|\|\dot{u}\| \\
& +\| \partial_{x} h\left(u, \operatorname{sat}^{*}(x)\right) \partial_{x} \operatorname{sat}^{*}(x) f\left(u, \operatorname{sat}^{*}(x)\right) \\
& \quad-\partial_{x} h\left(u, \operatorname{sat}^{*}(\hat{x})\right) \partial_{x} \operatorname{sat}^{*}(\hat{x}) f\left(u, \operatorname{sat}^{*}(\hat{x})\right) \| .
\end{aligned}
$$

Using the boundedness of $\dot{u}$ and the local Lipschitz continuity of $\partial_{u} h$, we can conclude, by the same argument as above, that the first term on the right-hand side of (A.1) is bounded by $\ell_{1}\|x-\hat{x}\|$ for some $\ell_{1}>0$. For the second term, let $F_{1}:=\partial_{x} h\left(u\right.$, sat $\left.{ }^{*}(x)\right), F_{2}:=\partial_{x} \operatorname{sat}^{*}(x)$, and $F_{3}:=$ $f\left(u\right.$, sat $\left.{ }^{*}(x)\right)$, and let $\hat{F}_{1}, \hat{F}_{2}$, and $\hat{F}_{3}$ denote the corresponding functions with argument $\hat{x}$. Noting that there is an $\ell_{2}>0$ such that for each $i \in 1, \ldots, 3,\left\|F_{i}\right\| \leq \ell_{2},\left\|\hat{F}_{i}\right\| \leq \ell_{2}$, and $\left\|F_{i}-\hat{F}_{i}\right\| \leq \ell_{2}\|x-\hat{x}\|$ (by the same type of argument as above), we can write $\left\|F_{1} F_{2} F_{3}-\hat{F}_{1} \hat{F}_{2} \hat{F}_{3}\right\|=\|\left(F_{1}-\hat{F}_{1}\right) F_{2} F_{3}+$ $\hat{F}_{1}\left(F_{2}-\hat{F}_{2}\right) F_{3}+\hat{F}_{1} \hat{F}_{2}\left(F_{3}-\hat{F}_{3}\right)\left\|\leq 3 \ell_{2}^{3}\right\| x-\hat{x} \|$. It now follows that Assumption 3 holds with $L_{2}=\ell_{1}+3 \ell_{2}^{3}$.

\section{B Proofs}

Proof (THEOREM 1) By a version of the bounded-real lemma (Saberi et al., 2006, Th. 11.45), the Hurwitz property of $\mathfrak{A}-K \mathfrak{C}$ and the bound $\|H(s)\|_{\infty}<\gamma$ implies that the LMI (14) with $X=P K$ is satisfied for some positive definite $P$. Define the Lyapunov function $W\left(t, \tilde{x}, \tilde{w}_{\mathrm{e}}\right)=\gamma V(t, \tilde{x})+$ $\tilde{w}_{\mathrm{e}}^{\prime} P \tilde{w}_{\mathrm{e}}$. Using Assumptions 1 and 2 we find that the derivative of $W$ along the trajectories of (12) satisfies

$$
\begin{aligned}
\dot{W}= & \gamma \frac{\partial V}{\partial t}(t, \tilde{x})+\gamma \frac{\partial V}{\partial \tilde{x}}(t, \tilde{x}) e(t, \tilde{x}) \\
& +\gamma \frac{\partial V}{\partial \tilde{x}}(t, \tilde{x})(g(u, \hat{x}, z)-g(u, \hat{x}, \hat{z})) \\
& +\tilde{w}_{\mathrm{e}}^{\prime}\left(P(\mathfrak{A}-K \mathfrak{C})+\left(\mathfrak{A}-K \mathfrak{C}^{\prime} P\right) \tilde{w}_{\mathrm{e}}+2 \tilde{w}_{\mathrm{e}}^{\prime} P \mathfrak{B}_{d} \tilde{d}\right. \\
\leq & -\gamma \alpha_{3}\|\tilde{x}\|^{2}+\gamma \alpha_{4} L_{1}\|\tilde{x}\|\|z-\hat{z}\| \\
& +\tilde{w}_{\mathrm{e}}^{\prime}\left(P \mathfrak{A}+\mathfrak{A}^{\prime} P-X \mathfrak{C}-\mathfrak{C}^{\prime} X^{\prime}\right) \tilde{w}_{\mathrm{e}}+2 \tilde{w}_{\mathrm{e}}^{\prime} P \mathfrak{B}_{d} \tilde{d} \\
\leq & -\gamma \alpha_{3}\|\tilde{x}\|^{2}+\gamma \alpha_{4} L_{1}\|\tilde{x}\|\left\|\tilde{w}_{\mathrm{e}}\right\|-\left\|\tilde{w}_{\mathrm{e}}\right\|^{2}+\gamma^{2}\|\tilde{d}\|^{2} \\
& +\left[\tilde{w}_{\mathrm{e}}^{\prime} \tilde{d}^{\prime}\right] T\left[\tilde{w}_{\mathrm{e}}^{\prime} \tilde{d}^{\prime}\right]^{\prime},
\end{aligned}
$$

where $T<0$ is the matrix on the left-hand side of (14). Using Assumption 3, we therefore have $\dot{W} \leq$ $-\gamma \alpha_{3}\|\tilde{x}\|^{2}+\gamma \alpha_{4} L_{1}\|\tilde{x}\|\left\|\tilde{w}_{\mathrm{e}}\right\|-\left\|\tilde{w}_{\mathrm{e}}\right\|^{2}+\gamma^{2} L_{2}^{2}\|\tilde{x}\|^{2}$. By studying the principal minors of the corresponding matrix quadratic form, we find that this expression is negative definite if $\gamma<4 \alpha_{3} /\left(4 L_{2}^{2}+\alpha_{4}^{2} L_{1}^{2}\right)$, and thus the result follows from the comparison lemma (Khalil, 2002, Lemma 3.4).

Proof (THEOREM 2) We start by showing that the pair $(\mathfrak{A}, \mathfrak{C})$ is detectable. Consider any eigenvalue $\lambda$ of $\mathfrak{A}$ that is unobservable with respect to the pair $(\mathfrak{A}, \mathfrak{C})$. There exist $w \in \mathbb{R}^{n_{w}}$ and $z \in \mathbb{R}^{p_{z}}$, not both zero, such that $(\mathfrak{A}-\lambda I)\left[\begin{array}{c}w \\ z\end{array}\right]=0$ and $\mathfrak{C}\left[\begin{array}{c}w \\ z\end{array}\right]=0$, which implies $(A-\lambda I) w+B_{z} z=0,-\lambda z=0$, and $C w+D_{z} z=0$. Clearly, either $z=0$ or $\lambda=0$. If $z=0$, then it follows that $w \neq 0$ and moreover, $(A-\lambda I) w=0$ and $C w=0$, which implies that $\lambda$ is an unobservable eigenvalue of the pair $(A, C)$. Since $(A, C)$ is a detectable pair, $\lambda$ must be in the open left-half complex plane. If $z \neq 0$, then $\lambda=0$, and we have $A w+B_{z} z=0$ and $C w+D_{z} z=0$, which implies that the Rosenbrock system matrix of the quadruple $\left(A, B_{z}, C, D_{z}\right)$ has rank less than $n_{w}+p_{z}$ for $\lambda=0$. The normal rank of the Rosenbrock matrix is $n_{w}+p_{z}$, which follows from leftinvertibility according to Saberi, Sannuti, and Chen (1995, Property 3.1.6). Hence, $\left(A, B_{z}, C, D_{z}\right)$ has an invariant zero at the origin, which contradicts Assumption 4. It follows that all unobservable eigenvalues of the pair $(\mathfrak{A}, \mathfrak{C})$ are in the open left-half complex plane, and hence it is detectable. Since the pair $(\mathfrak{A}, \mathfrak{C})$ is detectable, there exists a $K$ such that $\mathfrak{A}-K \mathfrak{C}$ is Hurwitz, which implies that $\|H(s)\|_{\infty}<\bar{\gamma}$ for some $\bar{\gamma}>0$. It follows that there exists a $\gamma^{*} \leq \bar{\gamma}$ such that $\|H(s)\|_{\infty}<\gamma$ can be achieved for all $\gamma>\gamma^{*}$. 
To prove the second statement of the theorem, we first show that when the quadruple $\left(A, B_{z}, C, D_{z}\right)$ is left-invertible and minimum-phase, the same holds for the triple $\left(\mathfrak{A}, \mathfrak{B}_{d}, \mathfrak{C}\right)$. This triple is obtained from $\left(A, B_{z}, C, D_{z}\right)$ by adding an integrator at each input point. Since integrators are leftinvertible, it follows from the definition of left-invertibility that $\left(\mathfrak{A}, \mathfrak{B}_{d}, \mathfrak{C}\right)$ is left-invertible. Let $\lambda$ be an invariant zero of $\left(\mathfrak{A}, \mathfrak{B}_{d}, \mathfrak{C}\right)$. Then the Rosenbrock system matrix corresponding to $\lambda$ is rank deficient, so there exist $w \in \mathbb{R}^{n_{w}}, z \in \mathbb{R}^{p_{z}}$, and $d \in \mathbb{R}^{p_{z}}$, not all zero, such that $(\mathfrak{A}-\lambda I)\left[\begin{array}{c}w \\ z\end{array}\right]+\mathfrak{B}_{d} d=0$ and $\mathfrak{C}\left[\begin{array}{l}w \\ z\end{array}\right]=0$, which implies that $(A-\lambda I) w+B_{z} z=0$, $-\lambda z+d=0$, and $C w+D_{z} z=0$. We must have $\left[w^{\prime}, z^{\prime}\right]^{\prime} \neq 0$, for if this were not the case, we would have $d \neq 0$ and $z=0$, which implies that $-\lambda z+d=d \neq 0$, which contradicts $-\lambda z+d=0$. From $(A-\lambda I) w+B_{z} z=0$ and $C w+D_{z} z=0$ we can therefore conclude, by the same argument as above, that $\lambda$ is an invariant zero of $\left(A, B_{z}, C, D_{z}\right)$. Hence $\lambda$ is in the open left-half complex plane, which shows that $\left(\mathfrak{A}, \mathfrak{B}_{d}, \mathfrak{C}\right)$ is minimum-phase.

The error dynamics (12a) is identical to the error dynamics of a strictly proper filter of the CSS architecture (see Saberi et al., 2006, eq. (9.12)), for a system $\dot{x}^{*}=\mathfrak{A} x^{*}+\mathfrak{B}_{d} u^{*}$, $y^{*}=\mathfrak{C} x^{*}$, where $x^{*}$ is the state to be estimated, $u^{*}$ is an unknown input, and $y^{*}$ is the available output. Hence, according to Saberi et al. (2006, Th. 9.22), $K$ can be chosen such that $\mathfrak{A}-K \mathfrak{C}$ is Hurwitz and $\|H(s)\|_{\infty}<\gamma$ for arbitrarily small $\gamma>0$ if the triple $\left(\mathfrak{A}, \mathfrak{B}_{d}, \mathfrak{C}\right)$ has no invariant zeros on the imaginary axis and the subspaces $\mathscr{S}^{-0}\left(\mathfrak{A}, \mathfrak{B}_{d}, \mathfrak{C}, 0\right)$ and $\mathscr{V}^{*}\left(\mathfrak{A}, \mathfrak{B}_{d}, \mathfrak{C}, 0\right)$ (Saberi et al., 2006, Ch. 3.2.5), intersect only at the origin. These conditions hold because $\left(\mathfrak{A}, \mathfrak{B}_{d}, \mathfrak{C}\right)$ is left-invertible and minimum-phase (see Saberi et al., 2006, Ch. 3.2.5), which completes the proof.

Proof (Theorem $\left.1^{\prime}\right)$ Let $W\left(t, \tilde{x}, \tilde{w}_{\mathrm{e}}\right)$ be defined in the same way as in the proof of Theorem 1. We then find that $\dot{W} \leq-\gamma \alpha_{3}\|\tilde{x}\|^{2}+\gamma \alpha_{4} L_{1}\|\tilde{x}\|\left\|\tilde{w}_{\mathrm{e}}\right\|-\left\|\tilde{w}_{\mathrm{e}}\right\|^{2}+\gamma^{2}\|\tilde{d}\|^{2}+$ $\left[\tilde{w}_{\mathrm{e}}^{\prime}, \tilde{d}^{\prime}\right] T\left[\tilde{w}_{\mathrm{e}}^{\prime}, \tilde{d}^{\prime}\right]^{\prime}$, where $T<0$ is the matrix on the left-hand side of (14). Using Assumption 3', we therefore have $\dot{W} \leq-\gamma \alpha_{3}\|\tilde{x}\|^{2}+\gamma \alpha_{4} L_{1}\|\tilde{x}\|\left\|\tilde{w}_{\mathrm{e}}\right\|-\left\|\tilde{w}_{\mathrm{e}}\right\|^{2}+$ $\gamma^{2} L_{2}^{2}\|\tilde{x}\|^{2}+\gamma^{2} L_{2}^{2}\left\|\tilde{w}_{\mathrm{e}}\right\|^{2}$. By studying the principal minors of the corresponding matrix quadratic form, we find that this expression is negative definite if $\gamma<\alpha_{3} / L_{2}^{2}$ and $\gamma<4\left(\alpha_{3}-\gamma L_{2}^{2}\right)\left(1-\gamma^{2} L_{2}^{2}\right) /\left(\alpha_{4}^{2} L_{1}^{2}\right)$. To see that the latter inequality can be satisfied, observe that the left-hand side decreases and the right-hand side increases as $\gamma \rightarrow 0$.

Proof (LEMMA 1) We have that $A_{\mathrm{n}}=R A_{\mathrm{b}}$, and we can therefore write $J=R A_{\mathrm{b}} A_{\mathrm{b}}^{\prime}-\hat{R} A_{\mathrm{b}} A_{\mathrm{b}}^{\prime}=\tilde{R} A_{\mathrm{b}} A_{\mathrm{b}}^{\prime}$. Define the Lyapunov function $V(\tilde{R})=\frac{1}{2}\|\tilde{R}\|^{2}$. Noting that $\operatorname{tr}(S(x) X)=$ 0 for any $x \in \mathbb{R}^{3}$ and symmetric $X \in \mathbb{R}^{3 \times 3}$, we have

$$
\begin{aligned}
\dot{V} & =\operatorname{tr}\left(\tilde{R}^{\prime}\left(\tilde{R} S\left(\omega^{\mathrm{b}}\right)-\Gamma \tilde{R} A_{\mathrm{b}} A_{\mathrm{b}}^{\prime}\right)\right) \\
& =\operatorname{tr}\left(\tilde{R}^{\prime} \tilde{R} S\left(\omega^{\mathrm{b}}\right)\right)-\operatorname{tr}\left(\tilde{R}^{\prime} \Gamma \tilde{R} A_{\mathrm{b}} A_{\mathrm{b}}^{\prime}\right) \\
& \leq-\lambda_{\min }\left(A_{\mathrm{b}} A_{\mathrm{b}}^{\prime}\right) \operatorname{tr}\left(\tilde{R}^{\prime} \Gamma \tilde{R}\right) \leq-\lambda_{\min }\left(A_{\mathrm{b}} A_{\mathrm{b}}^{\prime}\right) \lambda_{\min }(\Gamma)\|\tilde{R}\|^{2}
\end{aligned}
$$

(see Kleinman and Athans, 1968, for the relevant trace inequality). We can write $A_{\mathrm{b}}=Q \Lambda$, where the columns of $Q$ are the normalized columns of $A_{\mathrm{b}}$ and $\Lambda$ is a diagonal matrix with elements corresponding to the column norms of $A_{\mathrm{b}}$. Since $m^{\mathrm{b}}$ and $a^{\mathrm{b}}$ are non-parallel (i.e., $\left.\left\|m^{\mathrm{b}} \times a^{\mathrm{b}}\right\| \geq c_{\mathrm{obs}}>0\right)$, it follows that $Q$ is an orthogonal matrix. Thus $\lambda_{\min }\left(A_{\mathrm{b}} A_{\mathrm{b}}^{\prime}\right)=\lambda_{\min }\left(Q \Lambda^{2} Q^{\prime}\right)=\lambda_{\min }\left(\Lambda^{2}\right)=$ $\min \left\{\left\|m^{\mathrm{b}}\right\|,\left\|m^{\mathrm{b}} \times a^{\mathrm{b}}\right\|,\left\|m^{\mathrm{b}} \times\left(m^{\mathrm{b}} \times a^{\mathrm{b}}\right)\right\|\right\}^{2}=c^{2}$, where $c=\min \left\{\underline{m}, c_{\mathrm{obs}}, \underline{m} c_{\mathrm{obs}}\right\}>0$ (for $\underline{m}>0$ such that $\left\|m^{\mathrm{b}}\right\| \geq \underline{m}$ ). Thus, $\dot{V} \leq-\lambda_{\min }(\Gamma) c^{2}\|\tilde{R}\|^{2}$, and the result follows from the comparison lemma (Khalil, 2002, Lemma 3.4).

Proof (Lemma 2) Considering the function $V(\tilde{R})$ from the proof of Lemma 1 and noting that $V(\tilde{R})=\frac{1}{2} \tilde{x}^{\prime} \tilde{x}$ and $\dot{V} \leq-\lambda_{\min }(\Gamma) c^{2} \tilde{x}^{\prime} \tilde{x}$, we see that Assumption 1 is satisfied. We have $g(u, \hat{x}, z)=\operatorname{vec}\left(\Gamma J\left(m^{\mathrm{b}}, m^{\mathrm{n}}, a^{\mathrm{b}}, a^{\mathrm{n}}, \hat{R}\right)\right)$. Recalling that $z=a^{\mathrm{n}}$, it is therefore easy to confirm that $\| g(u, \hat{x}, z)-$ $g(u, \hat{x}, \hat{z})\|=\| \Gamma\left[0, m^{\mathrm{n}} \times(z-\hat{z}), m^{\mathrm{n}} \times\left(m^{\mathrm{n}} \times(z-\hat{z})\right)\right] A_{\mathrm{b}}^{\prime} \|$, and it follows from boundedness of $m^{\mathrm{n}}$ and $A_{\mathrm{b}}$ that Assumption 2 holds. We have $d(u, \dot{u}, x)=R \dot{a}^{\mathrm{b}}+R S\left(\omega^{\mathrm{b}}\right) a^{\mathrm{b}}$, and hence $\|d(u, \dot{u}, x)-d(u, \dot{u}, \hat{x})\|=\left\|\tilde{R}\left(\dot{a}^{\mathrm{b}}+S\left(\omega^{\mathrm{b}}\right) a^{\mathrm{b}}\right)\right\|$. Thus, Assumption 3 follows from boundedness of $\omega^{\mathrm{b}}, a^{\mathrm{b}}$, and $\dot{a}^{\mathrm{b}}$. It is easily verified that the $\Sigma_{2}$ subsystem is observable and that it has no invariant zeros at the origin, which implies that it is minimum-phase. Finally, the Rosenbrock system matrix has maximal rank for $\lambda=0$, which implies left-invertibility (see Moylan, 1977).

\section{References}

Açıkmeşe, B., Corless, M., 2011. Observers for systems with nonlinearities satisfying incremental quadratic constraints. Automatica 47, 1339-1348.

Arcak, M., Kokotović, P., 2001. Nonlinear observers: a circle criterion design and robustness analysis. Automatica 37 (12), 19231930.

Bornard, G., Hammouri, H., 2002. A graph approach to uniform observability of nonlinear multi output systems. In: Proc. IEEE Conf. Dec. Contr. Las Vegas, NV, pp. 701-706.

Brown, R. G., Hwang, P. Y. C., 1997. Introduction to Random Signals and Applied Kalman Filtering, 3rd Edition. Wiley, New York.

Chilali, M., Gahinet, P., 1996. $H_{\infty}$ design with pole placement constraints: An LMI approach. IEEE Trans. Automat. Contr. 41 (3), 358-367.

Crassidis, J. L., Markley, F. L., Cheng, Y., 2007. Survey of nonlinear attitude estimation methods. J. Guid. Contr. Dynam. 30 (1), 12-28.

Djurić, P. M., Kotecha, J. H., Zhang, J., Huang, Y., Ghirmai, T., Bugallo, M. F., Míguez, J., 2003. Particle filtering. IEEE Signal Process. Mag. 20 (5), 19-38.

Drakunov, S. V., 1992. Sliding-mode observers based on equivalent control method. In: Proc. IEEE Conf. Dec. Contr. Tucson, AZ, pp. 2368-2369.

Esfandiari, F., Khalil, H. K., 1987. Observer-based design of uncertain systems: Recovering state feedback robustness under matching conditions. In: Proc. Allerton Conf. Monticello, IL, pp. 97-106.

Fan, X., Arcak, M., 2003. Observer design for systems with multivariable monotone nonlinearities. Syst. Contr. Lett. 50 (4), 319-330.

Freidovich, L. B., Khalil, H. K., 2007. Lyapunov-based switching 
control of nonlinear systems using high-gain observers. Automatica 43 (1), 150-157.

Freidovich, L. B., Khalil, H. K., 2008. Performance recovery of feedback-linearization-based designs. IEEE Trans. Automat. Contr. 53 (10), 2324-2334.

Gauthier, J. P., Hammouri, H., Othman, S., 1992. A simple observer for nonlinear systems: Applications to bioreactors. IEEE Trans. Automat. Contr. 37 (6), 875-880.

Grip, H. F., Fossen, T. I., Johansen, T. A., Saberi, A., 2011a. Attitude estimation using biased gyro and vector measurements with time-varying reference vectors. IEEE Trans. Automat. Contr. (accepted).

Grip, H. F., Saberi, A., 2010. High-gain domination of nonlinear perturbations: Transformation to a canonical form by dynamic output shaping. In: Proc. IEEE Conf. Dec. Contr. Atlanta, GA, pp. 5043-5049.

Grip, H. F., Saberi, A., Johansen, T. A., 2011b. Estimation of states and parameters for linear systems with nonlinearly parameterized perturbations. Syst. Contr. Lett. 60 (9), 771-777.

Grip, H. F., Saberi, A., Johansen, T. A., 2011c. Observers for cascaded nonlinear and linear systems. In: Proc. IEEE Conf. Dec. Contr. Orlando, FL.

Hua, M.-D., 2010. Attitude estimation for accelerated vehicles using GPS/INS measurements. Contr. Eng. Pract. 18 (7), 723732 .

Julier, S. J., Uhlmann, J. K., 2004. Unscented filtering and nonlinear estimation. Proc. IEEE 92 (3), 401-422.

Kazantis, N., Kravaris, C., 1998. Nonlinear observer design using Lyapunov's auxiliary theorem. Syst. Contr. Lett. 34 (5), 241247.

Khalil, H. K., 2002. Nonlinear Systems, 3rd Edition. Prentice-Hall, Upper Saddle River, NJ.

Kleinman, D. L., Athans, M., 1968. The design of suboptimal linear time-varying systems. IEEE Trans. Automat. Contr. 13 (1968), 150-159.

Krener, A. J., Isidori, A., 1983. Linearization by output injection and nonlinear obervers. Syst. Contr. Lett. 3 (1), 47-52.

Marino, R., Tomei, P., 1995. Nonlinear Control Design: Geometric, Adaptive and Robust. Prentice-Hall, Upper Saddle River, NJ.

Maybeck, P. S., 1979. Stochastic Models, Estimation, and Control, Volume 1. Vol. 141 of Mathematics in Science and Engineering. Academic Press, New York.

Moraal, P. E., Grizzle, J. W., 1995. Observer design for nonlinear systems with discrete-time measurements. IEEE Trans. Automat. Contr. 40 (3), 395-404.

Moylan, P. J., 1977. Stable inversion of linear systems. IEEE Trans. Automat. Contr. 22 (1), 74-78.

Phanomchoeng, G., Rajamani, R., 2010. Observer design for Lipschitz nonlinear systems using Riccati equations. In: Proc. American Contr. Conf. Baltimore, MD, pp. 6060-6065.

Rajamani, R., 1998. Observers for Lipschitz nonlinear systems. IEEE Trans. Automat. Contr. 43 (3), 397-401.

Saberi, A., Sannuti, P., 1990. Observer design for loop transfer recovery and for uncertain dynamical systems. IEEE Trans. Automat. Contr. 35 (8), 878-897.

Saberi, A., Sannuti, P., Chen, B. M., 1995. $\mathrm{H}_{2}$ Optimal Control. Prentice-Hall, London.

Saberi, A., Stoorvogel, A. A., Sannuti, P., 2006. Filtering Theory. Birkhäuser, Boston.

Shim, H., Seo, J. H., 2003. Recursive nonlinear observer design: Beyond the uniform observability. IEEE Trans. Automat. Contr. 48 (2), 294-298.

Shuster, M. D., Oh, S. D., 1981. Three-axis attitude determination from vector observations. J. Guid. Contr. Dynam. 4 (1), 70-77.
Slotine, J.-J. E., Hedrick, J. K., Misawa, E. A., 1987. On sliding observers for nonlinear systems. J. Dyn. Syst. Meas. Contr. 109 (3), 245-252.

Thau, F. E., 1973. Observing the state of non-linear dynamic systems. Int. J. Contr. 17 (3), 471-479.

Vik, B., Fossen, T. I., 2001. A nonlinear observer for GPS and INS integration. In: Proc. IEEE Conf. Dec. Contr. Orlando, FL, pp. 2956-2961.

Zemouche, A., Boutayeb, M., Bara, G. I., 2008. Observers for a class of Lipschitz systems with extension to $H_{\infty}$ performance analysis. Syst. Contr. Lett. 57, 18-27. 